\title{
Casson fluid flow over a stretching surface with variable thermal conductivity and partial slip
}

\author{
Manish Raj ${ }^{1}$, AbhayKumar Jha ${ }^{*}$ and Anil Sharma ${ }^{3}$ \\ ${ }^{1,3}$ Department of Mathematics, University of RajasthanJaipur-302004, India \\ ${ }^{2}$ Department of Mathematics, JECRC University, Jaipur \\ ${ }^{1}$ E-mail: manishrajshekhu089@gmail.com ${ }^{2}$ \\ 22E-mail:itsabhay@rediffmail.com
}

\begin{abstract}
The flow and heat transfer of aCasson fluid flow over an impermeable stretching surface with variable thermal conductivity and non-uniform heat source/sink in the presence of partial slip is investigated. The resulting partial differential equations are reduced to a set of non-linear ordinary differential equation using similarity transformation and solved numerically using Runge-Kutta method along with shooting technique. The effects of the governing parameter on velocityand temperature fields are discussed.
\end{abstract}

Key words-Casson fluid, Partial slip, Variable thermal conductivity, Non-uniform heat source/sink

\section{INTRODUCTION}

The flow over a stretching sheet is significant due to its much application in engineering processes such as in the extraction of polymer sheets, paper production, wire drawing and glass-fiber production.Sakiadis [1,2] initiated the study of the boundary layer flow over a continuous solid surface moving with constant speed.The boundary layer problem considered by Sakiadis differs from the classical boundary-layer problem of Blasius [3], mainly due to the entrainment of the ambient liquid. Here the surface is assumed to be constant $\left(\mathrm{u}_{\mathrm{w}}=0\right)$ whereas most of the physical situations are concerned with extensible surface $\left(\mathrm{u}_{\mathrm{w}}=\mathrm{cx}\right)$ moving in a cooling liquid. Crane [4], for the first time, considered the boundary-layer behavior over an extensible surface, where he assumed the velocity of the surface to vary linearly with the distance from the slit. Carrayher and Crane [5] analyzed the heat transfer due to a continuous stretching sheet. The pioneering work of Crane was extended by many authors Gupta and Gupta [6], Grabka and Babba [7], Chen and Chur [8], and Chaim [9].

In engineering applications, homogeneous or heterogeneous reactions often lead to a significant heat release accompanied by non-isothermal conditions that require the introduction of a heat source/sink term in the energy equation.Cartel [10-12] studied the flow and heat transfer characteristics with linearly and Non-linearly stretching sheet for both Newtonian and Non-Newtonian fluids with internal heat generation/absorption and suction/injection. The study of flow and heat transfer for electrically conducting fluids under the influence of a magnetic field has attracted the interest of many investigators. MHD flows have great significance for the application in the field of satellite and planetary magnetospheres, aeronautics and chemical engineering. Sarpakaya [13] was the first to study the MHD effects on the flow of a non-Newtonian fluid. Pal and Mondal $[14,15]$ considered the MHD fluid for their study. The MHD fluids have been considered by many researchers [16-21,41]. Abel et al. [22] studied the effect of variable viscosity on the heat transfer of viscoelastic fluid due to stretching sheet. Vajravelu and Rollins [23] and Vajravelu and Nayfeh [24] have studied the effect of a uniform heat source/sink on the heat transfer from a stretching sheet. Bhattacharya and Vajravelu [26] studied the stagnation-point flow over an exponential stretching sheet. Abo-Eldahub and Elaziz [27] investigated heat transfer considering a non-uniform heat source/sink. Recent work of Nandep-Panavar et al. [28], Abel et al. [29] and Bataller [30] in the case of a visco elastic liquid flow due to a stretching sheet.

The no-slip boundary condition is known as the central tenet of the Navier-Stokes theory. But there are situations wherein such a condition is not approximate. Especially the no-slip condition is insufficient for the most non-Newtonian liquids. The liquids exhibiting boundary slip find applications in technology such as polishing of artificial heart valves and internal cavities. Navier [31] suggested a slip boundary condition in terms of shear stress. The work of Navier was extended by many authors [32,40,43,44]. Mustafa et al. [45] studied the effect of non-uniform heat source on heat transfer of non-Newtonian power law fluid over a non-linear stretching sheet. John and Kumaran studied the heat transfer due to a heat source in MHD unsteady stretching sheet flow. Mahantish M. et al. [47] studied the MHD flow and heat transfer over a stretching surface with variable thermal conductivity and partial slip. In the above work, theCasson fluid flow over a stretching surface with variable thermal conductivity and partial slip has not investigated yet. 


\section{FORMATION OF THE PROBLEM}

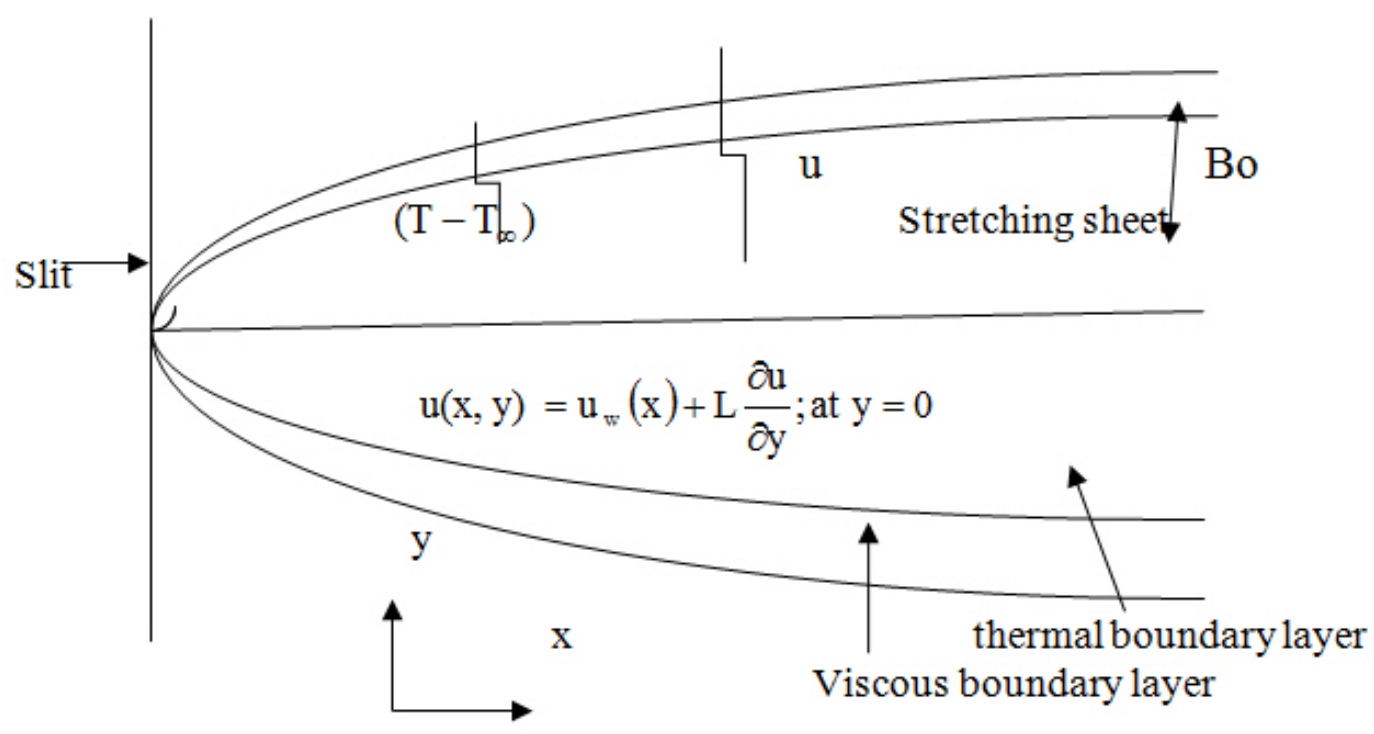

Fig 1 schematic of a stretching sheet problem with partial slip condition

Consider a steady, laminar, two dimensional flow of an incompressible viscous liquid past a flat impermeable sheet coinciding with the plane $y=0$ and the flow being confined to upper half of the plane $(y>0)$. The flow is generated, due to stretching of the sheet, caused by simultaneous application of two equal and opposite forces along the $\mathrm{x}$-axis (see Fig. 1) the sheet is stretched keeping the origin fixed, with a velocity varying linearly with the distance from the slit. Let $\mathrm{B}_{0}$ be the externally applied magnetic field. Since the magnetic Reynolds number is assumed to be small, the induced magnetic field is negligible. We take the $\mathrm{x}$-axis along the surface and $\mathrm{y}$-axis is normal to it.

We assume that the rheological equation of state for an isotropic and incompressible flow of a Casson fluid can be written as

$$
\tau_{\mathrm{ij}}= \begin{cases}\left(\mu_{\mathrm{B}}+\tau_{\mathrm{y}} / \sqrt{2 \pi}\right) 2 \mathrm{e}_{\mathrm{ij}} & \pi>\pi \mathrm{c} \\ \left.\overline{(\mu}_{\mathrm{B}}+\tau_{\mathrm{y}} / \sqrt{2 \pi \mathrm{c}}\right) 2 \mathrm{e}_{\mathrm{ij}} & \pi<\pi \mathrm{c}\end{cases}
$$

Where $\mu_{\mathrm{B}}$ is plastic dynamics viscosity of the Casson fluid, $\tau_{\mathrm{y}}$ is the yield stress of fluid, $\pi$ is the product of component of deformation rate with itself, namely, $\pi=\mathrm{e}_{\mathrm{ij}} \mathrm{e}_{\mathrm{ij}}, \mathrm{e}_{\mathrm{ij}}$ is the $(\mathrm{i}, \mathrm{j})$ the component of the deformation rate of $\pi_{\mathrm{c}}$ is the critical value $\pi$. The momentum, temperature and concentration equation under the above assumptions are written as

$$
\frac{\partial \mathrm{u}}{\partial \mathrm{x}}+\frac{\partial \mathrm{v}}{\partial \mathrm{y}}=0
$$

$\mathrm{u} \frac{\partial \mathrm{u}}{\partial \mathrm{x}}+\mathrm{v} \frac{\partial \mathrm{u}}{\partial \mathrm{y}}=-\frac{1}{\rho} \frac{\partial \mathrm{p}}{\partial \mathrm{x}}+\mathrm{v}\left(1+\frac{1}{\beta}\right)\left(\frac{\partial^{2} \mathrm{u}}{\partial \mathrm{x}^{2}}+\frac{\partial^{2} \mathrm{u}}{\partial \mathrm{y}^{2}}\right)-\frac{\sigma \mathrm{B}_{0}}{\rho} \mathrm{u}$

$\mathrm{u} \frac{\partial \mathrm{v}}{\partial \mathrm{x}}+\mathrm{v} \frac{\partial \mathrm{v}}{\partial \mathrm{y}}=-\frac{1}{\rho} \frac{\partial \mathrm{p}}{\partial \mathrm{y}}+\mathrm{v}\left(1+\frac{1}{\beta}\right)\left(\frac{\partial^{2} \mathrm{v}}{\partial \mathrm{x}^{2}}+\frac{\partial^{2} \mathrm{v}}{\partial \mathrm{y}^{2}}\right)$

$\left(\mathrm{u} \frac{\partial \mathrm{T}}{\partial \mathrm{x}}+\mathrm{v} \frac{\partial \mathrm{T}}{\partial \mathrm{y}}\right)=\frac{1}{\rho \mathrm{C}_{\mathrm{p}}} \frac{\partial}{\partial \mathrm{y}}\left(\mathrm{k} \frac{\partial \mathrm{T}}{\partial \mathrm{y}}\right)+\frac{1}{\rho \mathrm{C}_{\mathrm{p}}}\left(\frac{\mathrm{ku}_{\mathrm{w}}(\mathrm{x})}{\mathrm{xv}}\right)\left[\mathrm{A}^{*}\left(\mathrm{~T}_{\mathrm{w}}-\mathrm{T}_{\infty}\right) \mathrm{e}^{-\mathrm{S} \eta}+\mathrm{B}^{*}\left(\mathrm{~T}-\mathrm{T}_{\infty}\right)\right]$ 
Where $u$ and $v$ are the velocity components along $\mathrm{x}$ - and $\mathrm{y}$ direction, respectively, $v$ is the kinematic viscosity, $\rho$ is the density and $\sigma$ is the electric conductivity. $\mathrm{T}$ is the temperature of the liquid, $\mathrm{k}$ is the thermal conductivity, $\mathrm{C}_{\mathrm{p}}$ is the specific heat at constant, constant pressure.

The second term on the right hand side of equation (4) represent the non-uniform heat source/sink term. $\mathrm{A}^{*}$ and $\mathrm{B}^{*}$ are the parameters of the space and temperature-dependent internal heat generation/absorption. The case $\mathrm{A}^{*}$ $>0$ and $\mathrm{B}^{*}>0$ corresponds to internal heat generation, while the case $\mathrm{A}^{*}<0, \mathrm{~B}^{*}<0$ corresponds to internal heat absorption and $\mathrm{k}=\mathrm{k}_{\infty}\left[1+\varepsilon\left(\frac{\mathrm{T}-\mathrm{T}_{\infty}}{\mathrm{T}_{\mathrm{w}}-\mathrm{T}_{\infty}}\right)\right]$ when $\varepsilon$ is small parameter.

The boundary conditions are

$$
\begin{aligned}
& \left.\begin{array}{l}
\mathrm{u}(\mathrm{x}, \mathrm{y})=\mathrm{cx}+\mathrm{L} \frac{\partial \mathrm{u}}{\partial \mathrm{y}} ; \mathrm{v}=0 ; \mathrm{p}=\mathrm{p}_{\mathrm{w}} \text { at } \mathrm{y}=0 \\
\mathrm{u} \rightarrow 0 \quad \text { as } \mathrm{y} \rightarrow \infty \\
\mathrm{T}=\mathrm{T}_{\mathrm{w}}=\mathrm{T}_{\infty}+\mathrm{A}_{0}\left(\frac{\mathrm{x}}{\mathrm{L}}\right)^{\lambda} \text { at } \mathrm{y}=0 \\
\mathrm{~T} \rightarrow \mathrm{T}_{\infty} \quad \text { as } \mathrm{y} \rightarrow 0
\end{array}\right\}[\text { P.S.T case }]
\end{aligned}
$$

Where $A_{0}$ is the constant; $\lambda$ is the variable wall-temperature parameter; $T_{w}$ is wall temperature. $T_{\infty}$ the temperature of liquid far away from sheet (in this problem we take $\lambda=1$ ]

$$
\left.\begin{array}{l}
-\mathrm{k} \frac{\partial \mathrm{T}}{\partial \mathrm{y}}=\mathrm{q}_{\mathrm{w}}=\mathrm{D}\left(\frac{\mathrm{x}}{\mathrm{L}}\right)^{\lambda} \text { at } \mathrm{y}=0 \\
\mathrm{~T} \rightarrow \mathrm{T}_{\infty} \text { as } \mathrm{y} \rightarrow \infty
\end{array}\right\} \text { [PHF Case] }
$$

Where $\mathrm{D}$ is positive constant; $\mathrm{L}$ is the characteristic length.

\section{ANALYSIS}

The continuity equation is identically satisfied by stream function $\psi(\mathrm{x}, \mathrm{y})$

given as

$$
\mathrm{u}=\frac{\partial \psi}{\partial \mathrm{y}} ; \mathrm{v}=-\frac{\partial \psi}{\partial \mathrm{x}}
$$

In the solution of equation (2), (3) and (4), the following dimensionless variables are derived as

$$
\begin{aligned}
& \psi=(\mathrm{c} v)^{1 / 2} \mathrm{xf}(\eta) ; \eta=\left(\frac{\mathrm{c}}{\mathrm{v}}\right)^{1 / 2} \mathrm{y} \\
& \frac{\mathrm{p}}{\rho c \nu}=\frac{\mathrm{P}_{\mathrm{w}}}{\rho c \nu}-\frac{1}{2} \mathrm{~h}(\eta) ; \theta(\eta)=\frac{\mathrm{T}-\mathrm{T}_{\infty}}{\mathrm{T}_{\mathrm{w}}-\mathrm{T}_{\infty}} \\
& \mathrm{P}=\mathrm{P}_{\mathrm{w}}-\frac{1}{2} \mathrm{~h}(\eta)
\end{aligned}
$$

Solution of equation (2), (3) and (4) with help of equation (9), (10) and (11) can be written as

$$
\left(1+\frac{1}{\beta}\right) \mathrm{f}^{\prime \prime}+\mathrm{f} \mathrm{f}^{\prime \prime}-\mathrm{f}^{2}-\mathrm{Mn} \mathrm{f}^{\prime}=0
$$




$$
\begin{gathered}
\left(1+\frac{1}{\beta}\right) \mathrm{f}^{\prime \prime}+\mathrm{ff}^{\prime}-\frac{1}{2} \mathrm{~h}^{\prime}=0 \\
(1+\varepsilon \theta) \theta^{\prime \prime}+\operatorname{Pr}\left(\mathrm{f} \theta^{\prime}-\mathrm{f}^{\prime} \theta\right)+\varepsilon \theta^{\prime 2}+(1+\varepsilon \theta)\left(\mathrm{A}^{*} \mathrm{f}^{\prime}+\mathrm{B}^{*} \theta\right)=0
\end{gathered}
$$

and boundary condition (5) -(8) take the form

$$
\begin{aligned}
& \left.\begin{array}{l}
\mathrm{f}(\eta)=0 ; \mathrm{f}^{\prime}(\eta)=1+\gamma \mathrm{f}^{\prime \prime}(\eta) ; \mathrm{h}(\eta)=0 \text { at } \eta=0 \\
\mathrm{f}^{\prime}(\eta) \rightarrow 0 \text { as } \eta \rightarrow \infty \\
\left.\begin{array}{l}
\theta(\eta)=1 \text { at } \eta=0 \\
\theta(\eta) \rightarrow 0 \text { at } \eta \rightarrow \infty
\end{array}\right\}(\mathrm{PST}) \\
\theta^{\prime}(\eta)=-\frac{1}{1+\varepsilon \theta} \text { at } \eta=0 \\
\theta(\eta) \rightarrow 0 \text { as } \eta \rightarrow \infty
\end{array}\right\}(\text { PHT) }
\end{aligned}
$$

where

$$
M_{n}=\frac{\sigma B_{0}}{\rho c} \quad \text { (Magnetic parameter) }
$$

$\gamma=\mathrm{L} \sqrt{\frac{\mathrm{C}}{\mathrm{v}}} \quad$ (slip parameter; $\mathrm{L}$ is denoted the slip length)

$$
\begin{aligned}
& P_{w}=\frac{P_{w}}{\rho c v} \quad \text { (dimensionless pressure distribution at wall which is } \\
& \quad \text { very negligible) } \\
& \beta=\mu_{\beta} \sqrt{2 \pi_{c} / \tau_{y}} \quad \text { (Casson parameter) }
\end{aligned}
$$

\section{RESILT and DISCUSSION}

The set of non-linear ordinary differential equation (12), (13) and (14) with boundary conditions (15) - (18) were solved numerically using Runge-Kutta fourth order method. We have compared our results with Mahantesh M. [47] in table 1 and table 2 and it is observed that results are in very good concord.

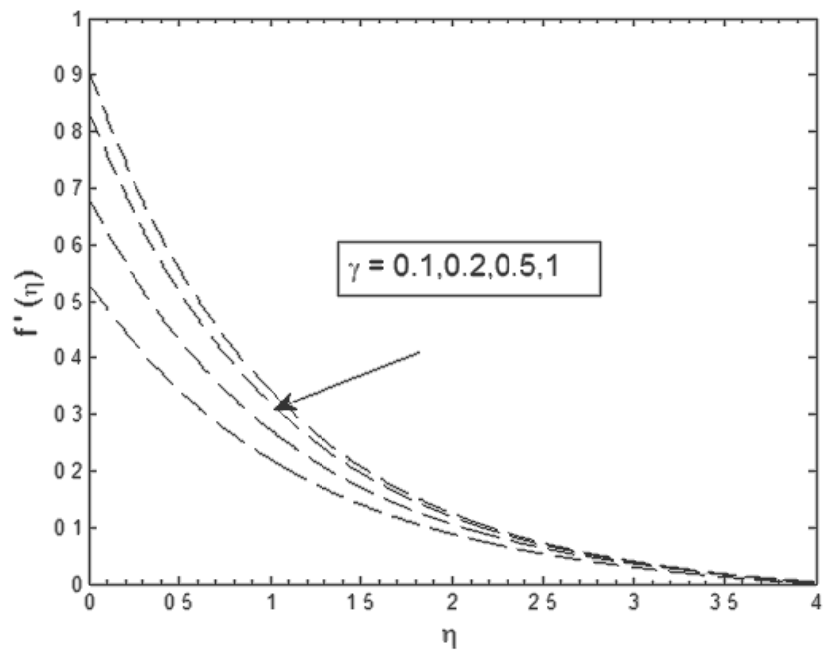

Figure 2 Effect of slip parameter $\gamma$ on axial velocity profiles with $\mathrm{Mn}=1$ and $\beta=\infty$ 


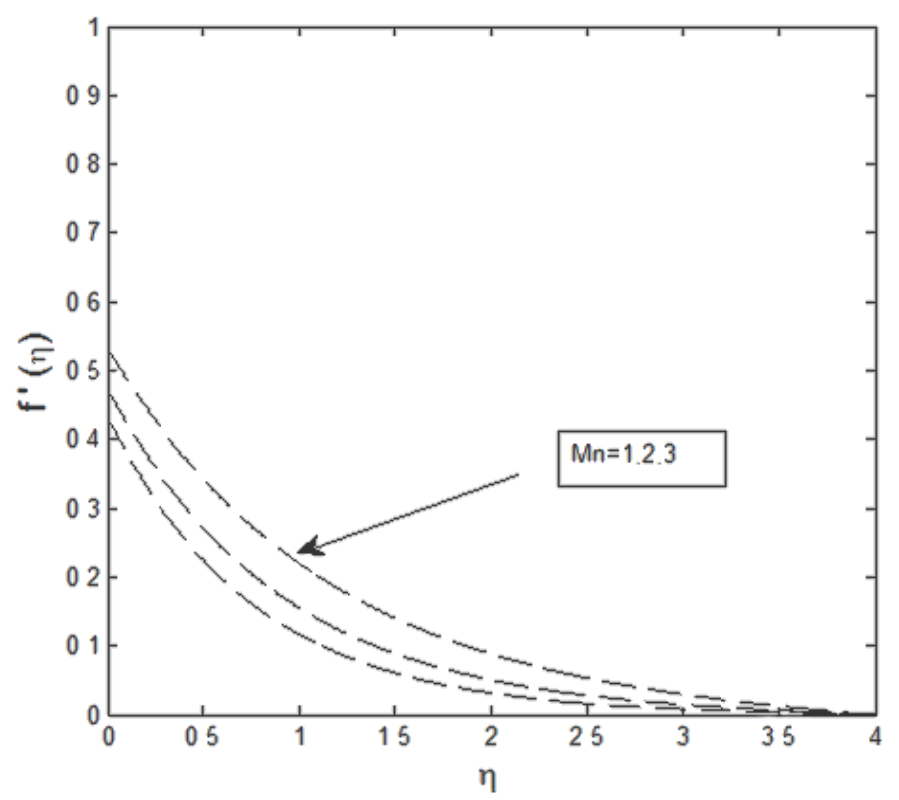

Fig.3 Effect of magnetic parameter Mn on axial velocity profile with slip parameter $\gamma=1$ and $\beta=\infty$

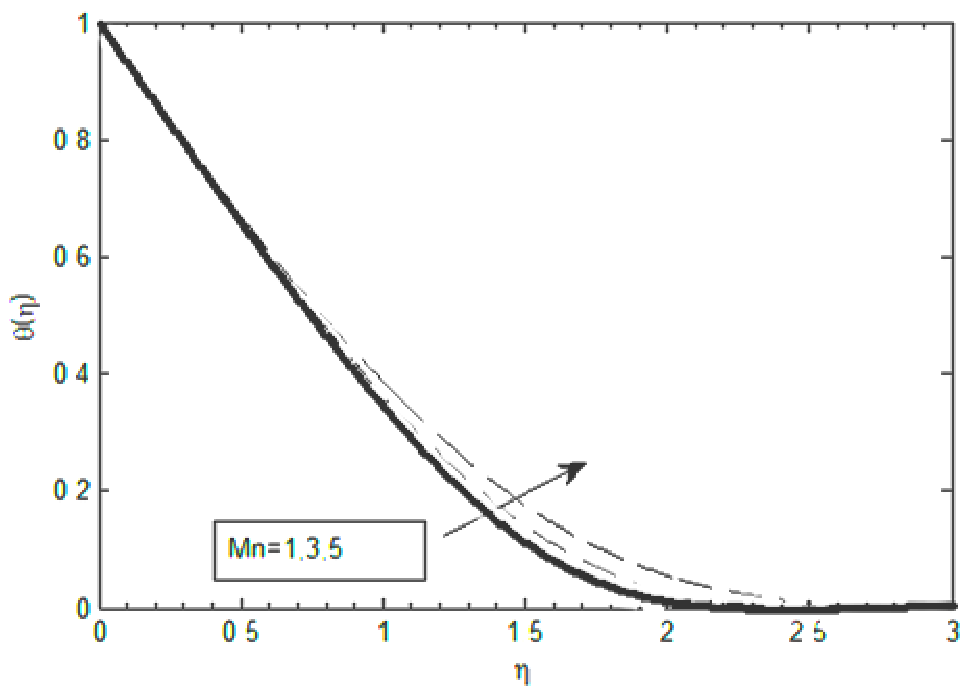

(a)

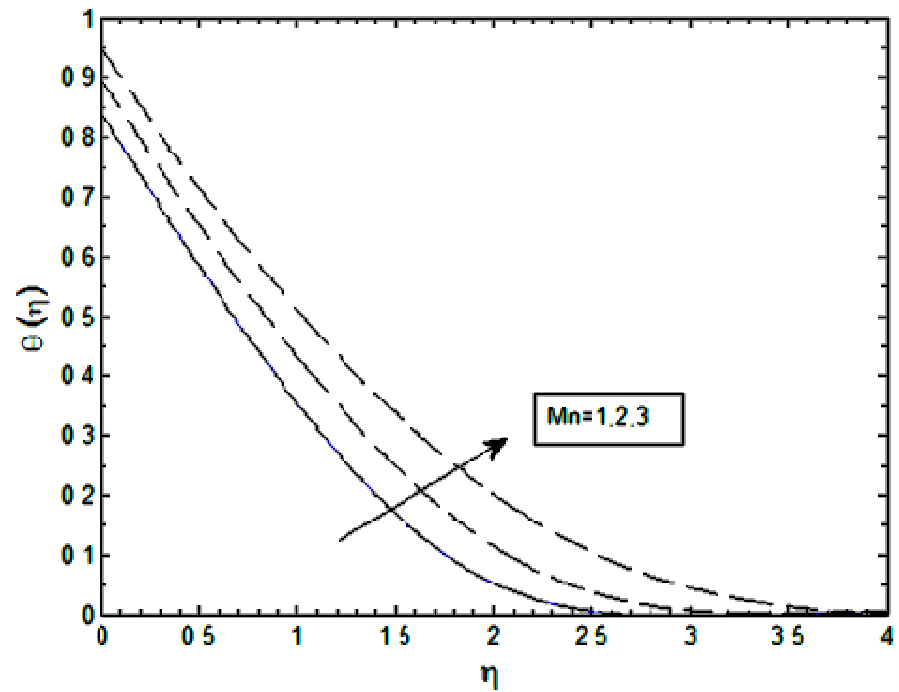

(b)

Fig 4 Effect of magnetic parameter Mn (a) PST case and (b)PHF case on temperature profiles at $\operatorname{Pr}=1, \mathrm{~A}^{*}=-0.01, \mathrm{~B}^{*}=-0.01$ and $\mathrm{e}=1$, and $\gamma$ $=1$ 


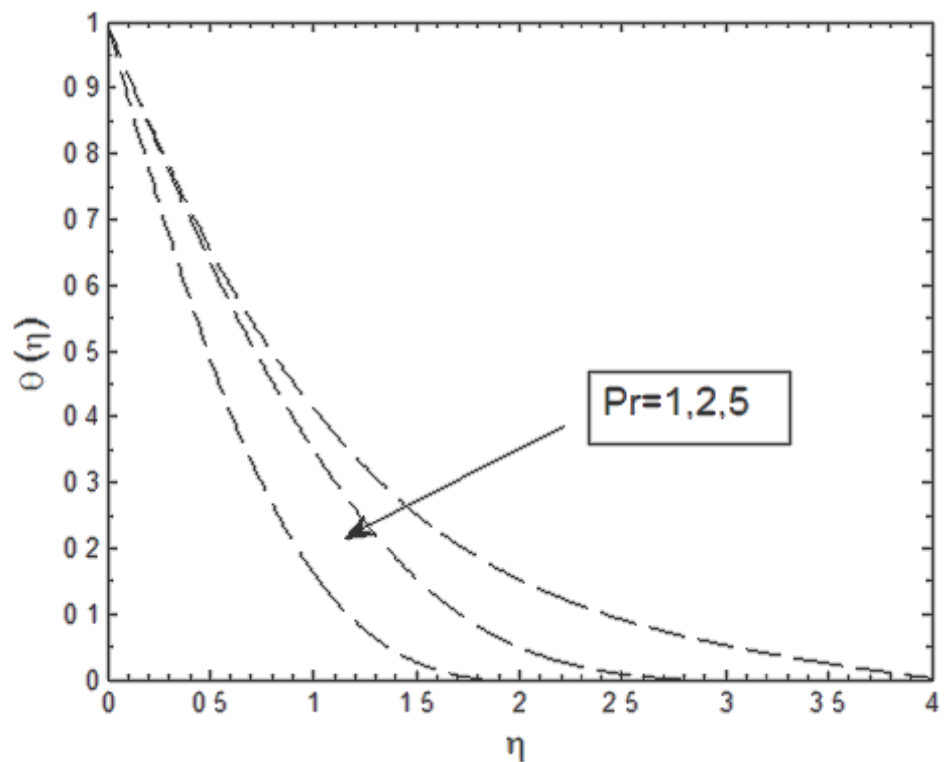

(a)

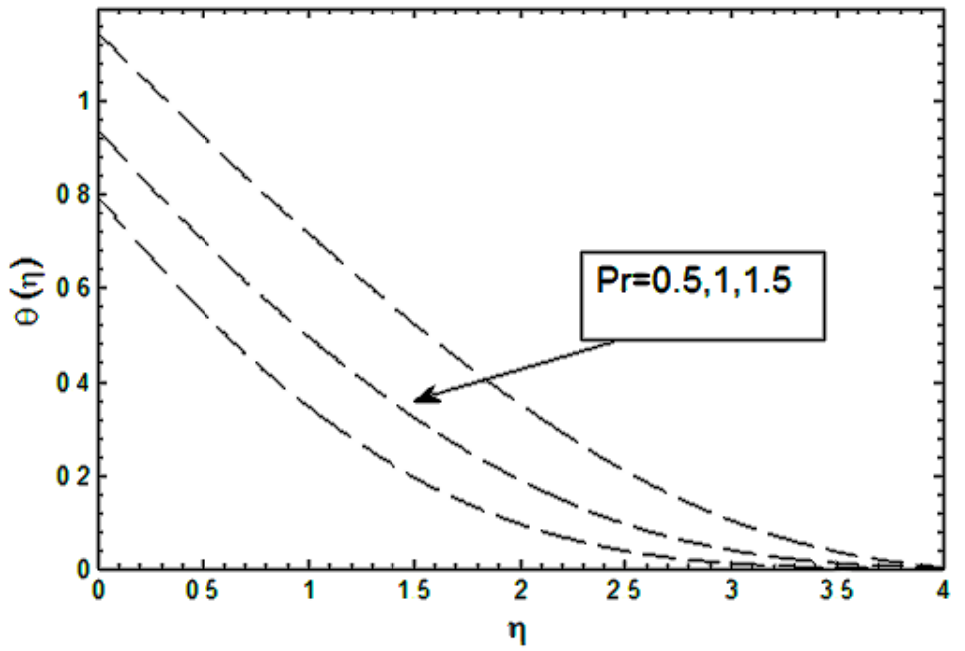

(b)

Fig 5.Effect of magnetic parameter $\operatorname{Pr}$ on temperature profiles at $\mathrm{Mn}=1, \mathrm{~A}^{*}=-0.01, \mathrm{~B}^{*}=-0.01$ and $\mathrm{e}=1$, and $\gamma=\mathbf{1}$ 


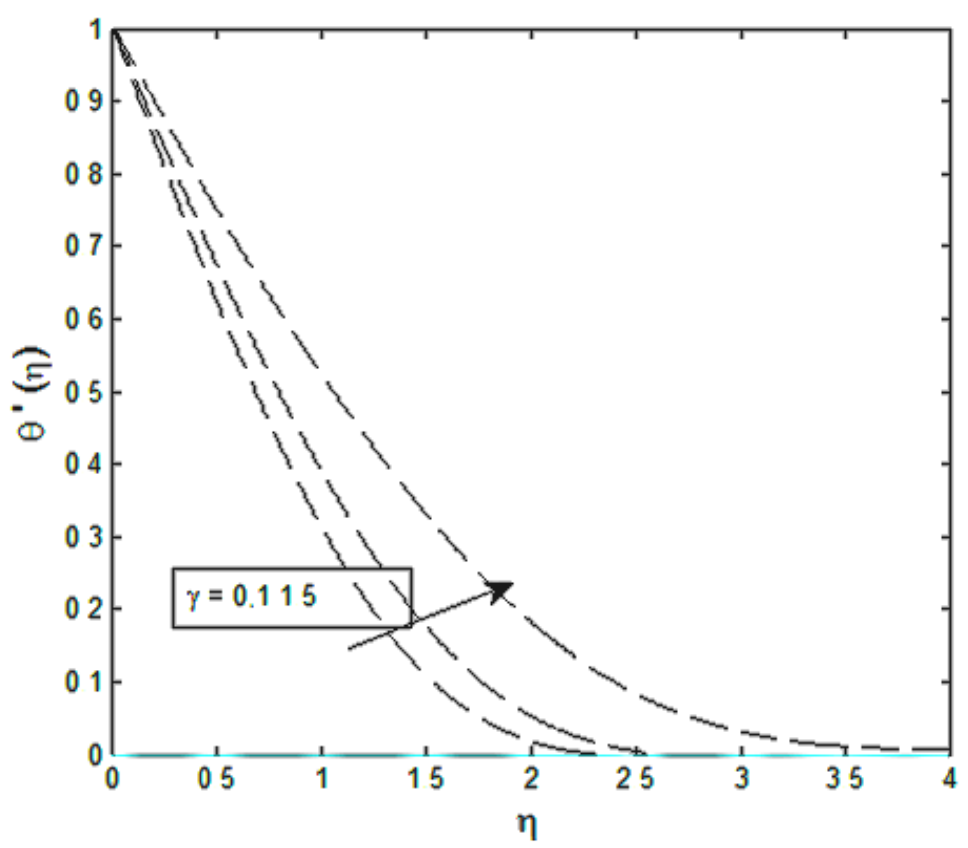

(a)

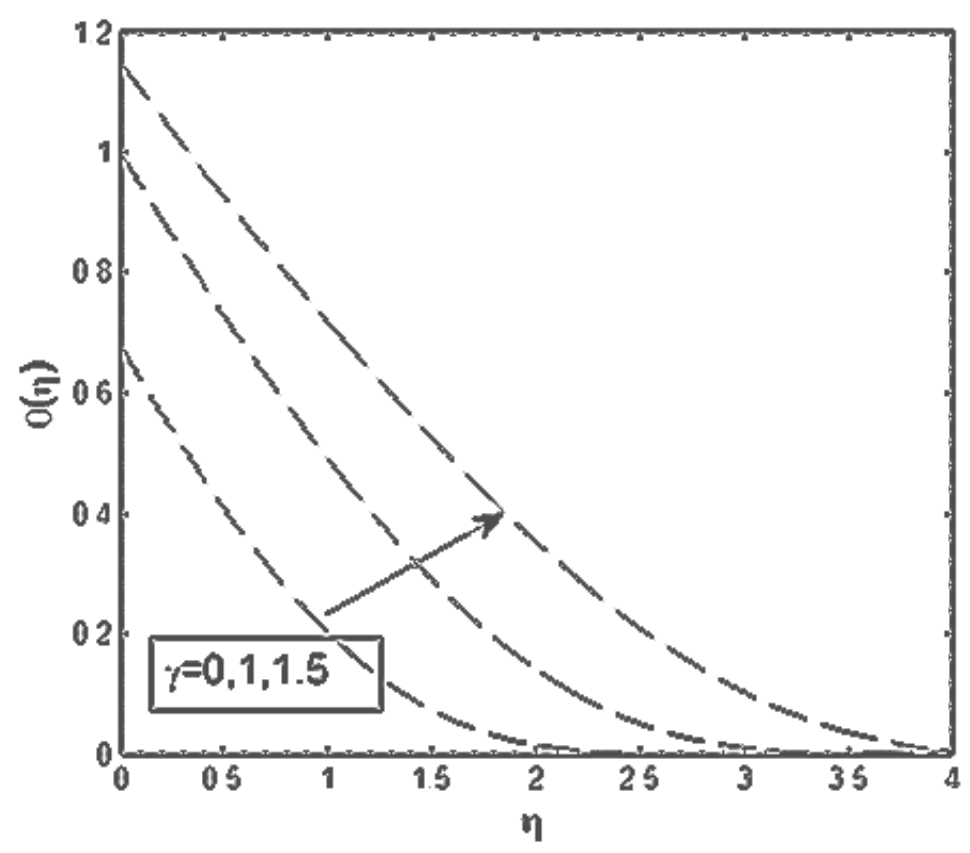

(b)

Fig 6 Effect of slip parameter $\gamma$ (a) PST case (b) PHF case on temperature profiles at $\operatorname{Pr}=1, \mathrm{Mn}=1, \mathrm{~A}^{*}=-0.01, \mathrm{~B}^{*}=-0.01$ and $\mathrm{e}=1$ 


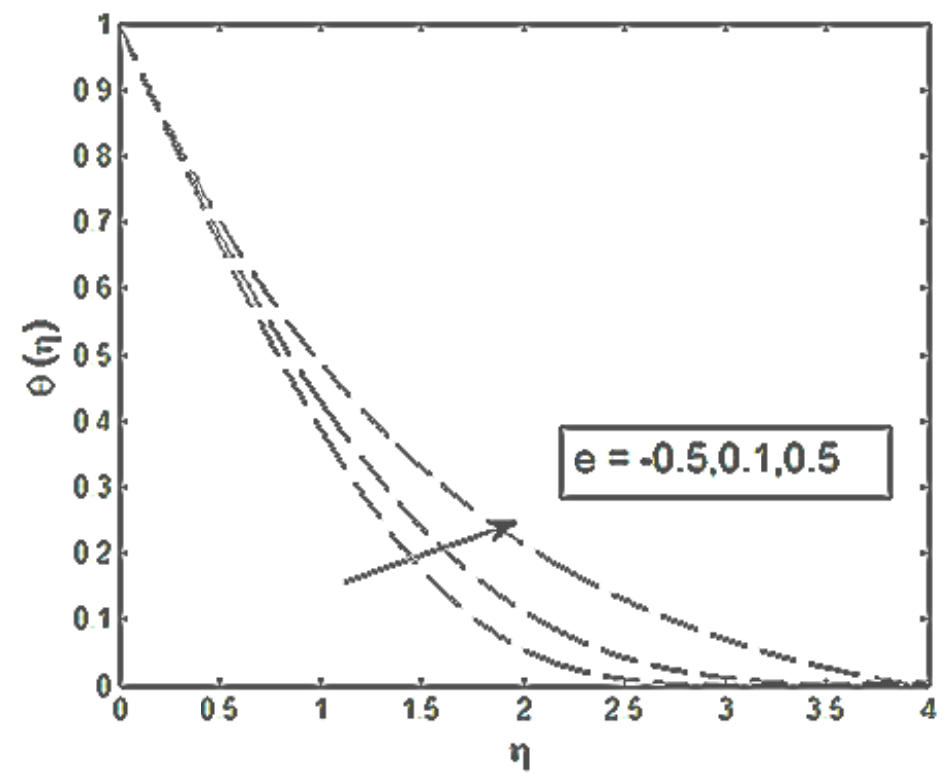

(a)

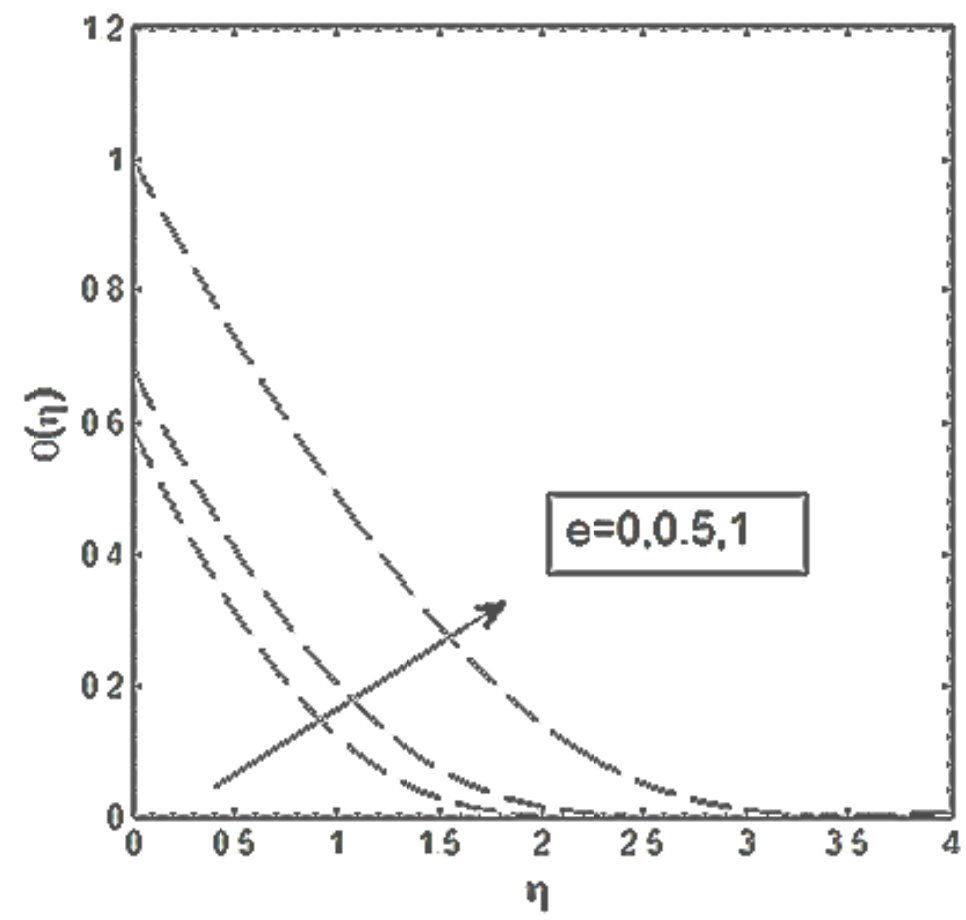

(b)

Fig 7.Effect of variable thermal conductivity (a)PST case(b) PHF case on temperature profiles at $\mathrm{Mn}=1, \mathrm{~A}^{*}=-0.01, \mathrm{~B}^{*}=-0.01$ and $\mathrm{Pr}=1$, and $\gamma=1$ 


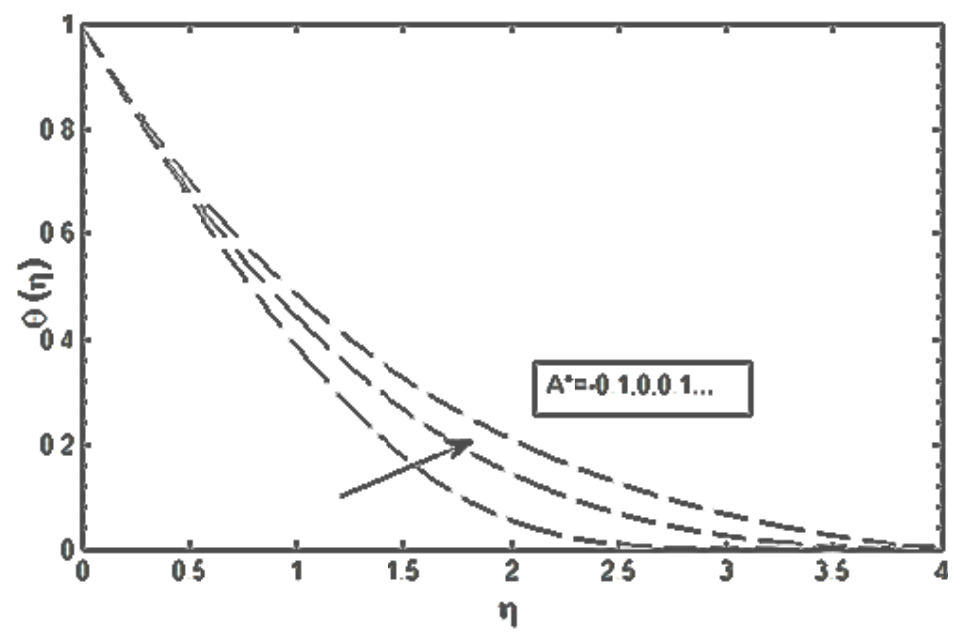

(a)

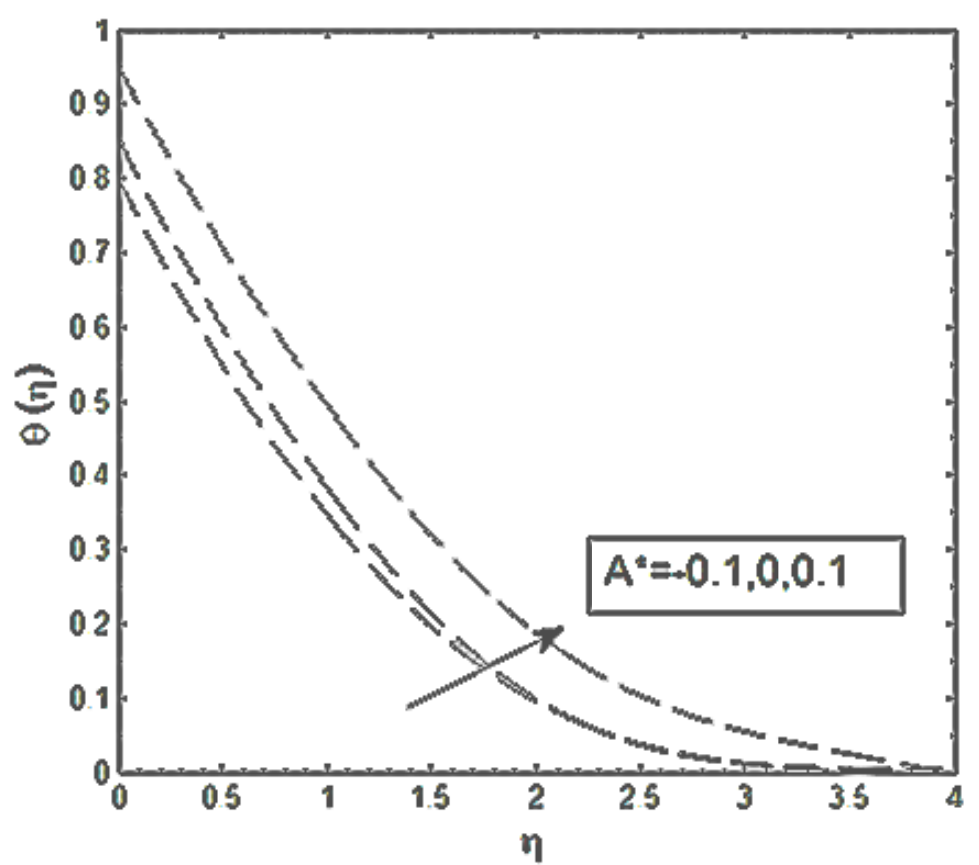

(b)

Fig 8.Effect of space -dependent heat source/sink parameter A*(a)PST case (b)PHF case on temperature profiles at Mn=1, $\mathrm{B}^{*}=-0.01$ $\mathrm{e}=1$, and $\operatorname{Pr}=1$, and $\gamma=\mathbf{1}$ 


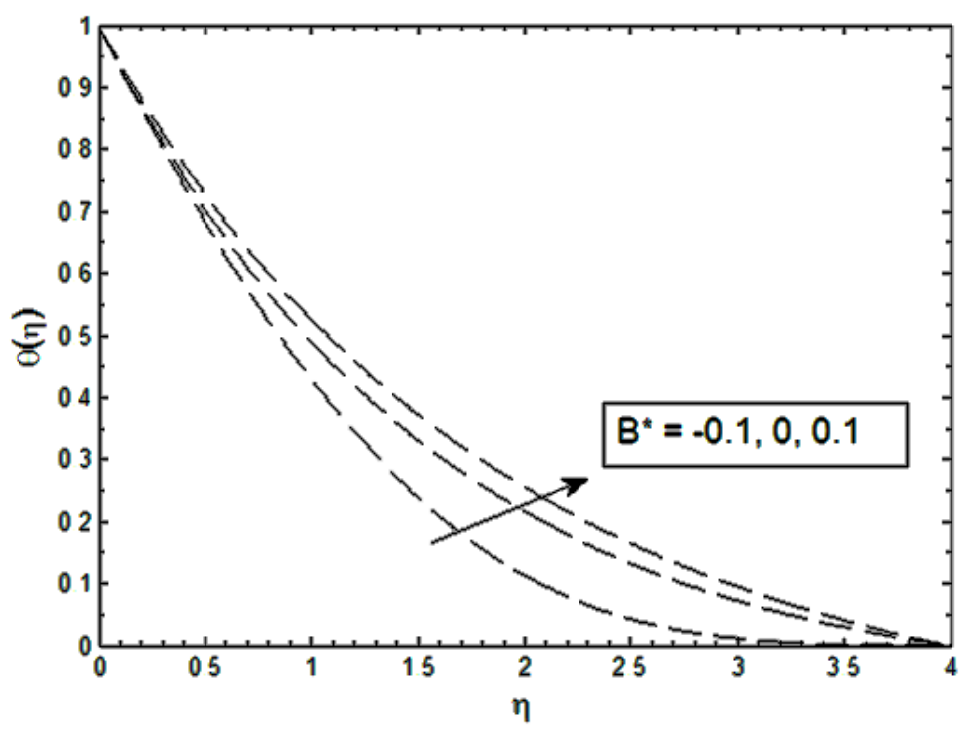

(a)

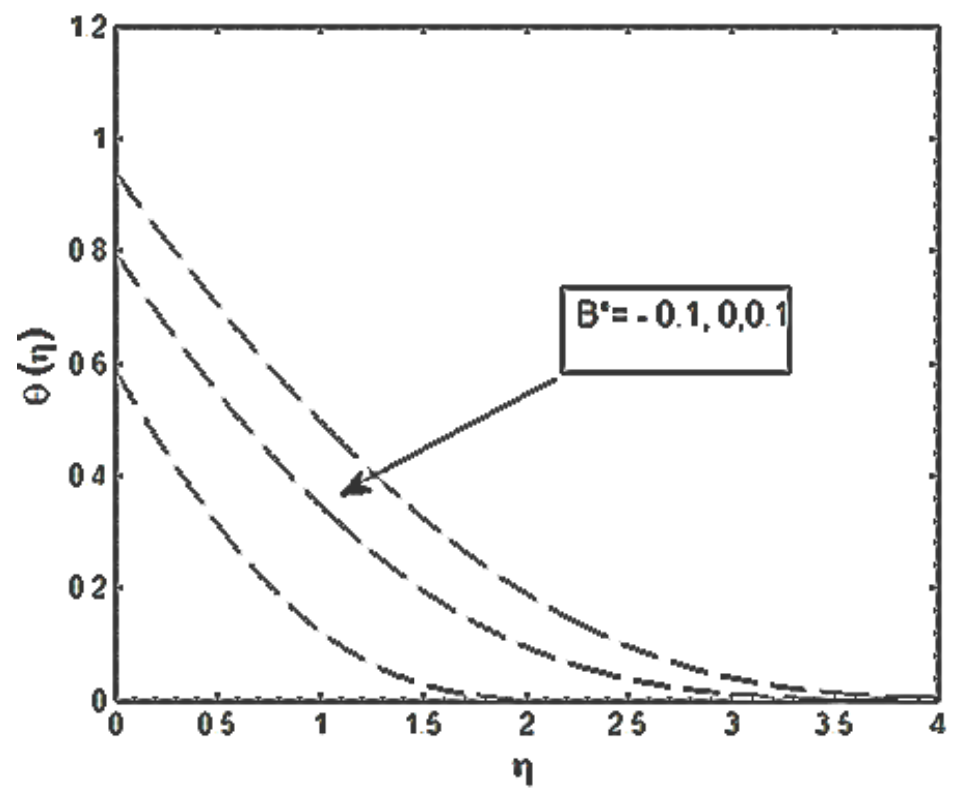

(b)

Fig 9.Effect of temperature -dependent heat source/sink parameter B* (a)PST case (b)PHF case on temperature profiles at Mn=1,A*=-0.01 $\mathrm{e}=1$, and $\operatorname{Pr}=1$, and $\gamma=1$ 


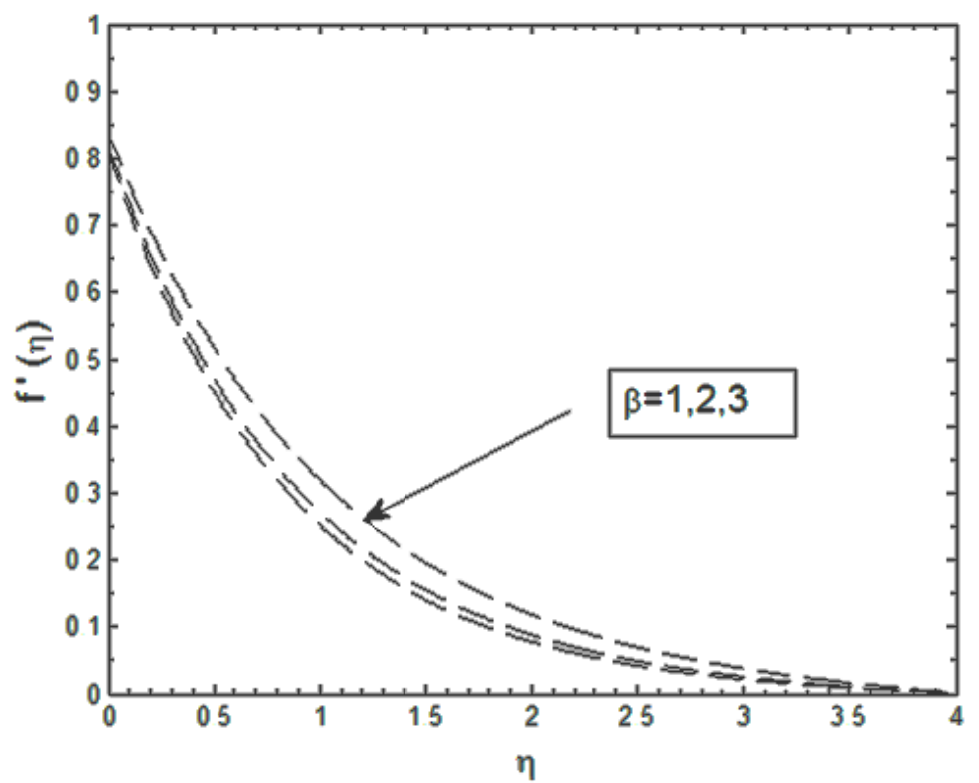

Fig 10.Effect of Casson parameter $\beta$ on velocity profiles at $\mathrm{Mn}=1, \mathrm{~B}^{*}=-0.01, \mathrm{~A}^{*}=-0.01 \mathrm{e}=1$, and $\mathrm{Pr}=1$, and $\gamma=\mathbf{1}$

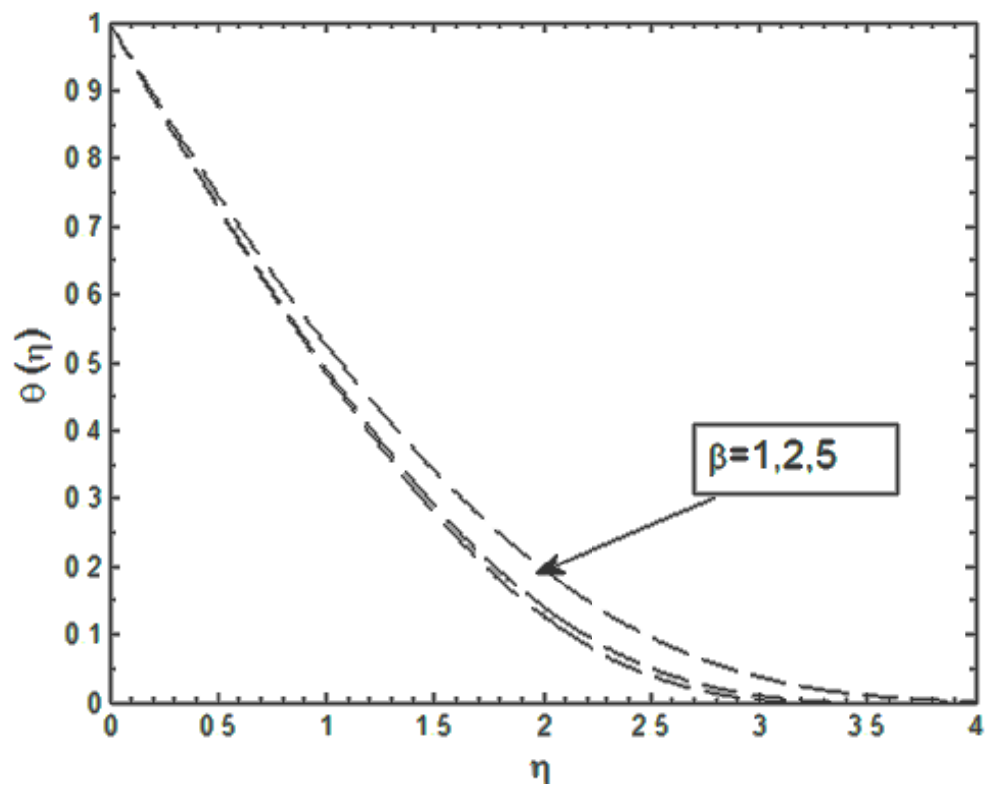

(a) 


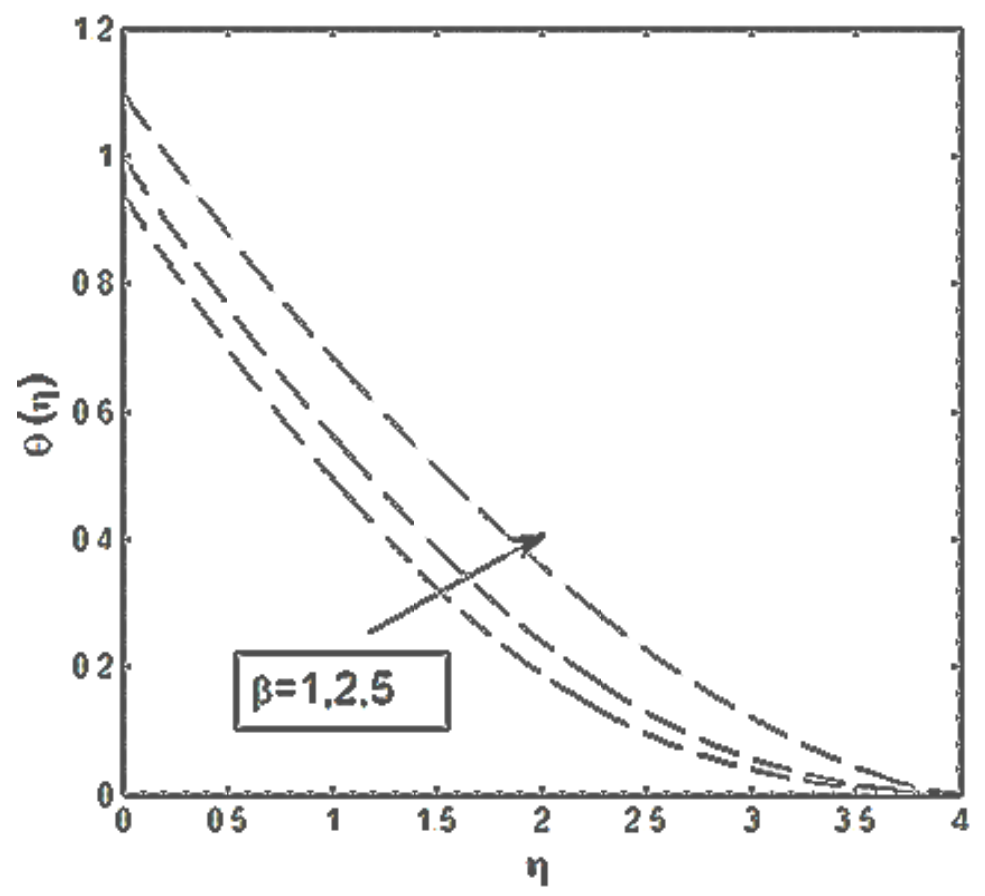

(b)

Fig11...Effect of Casson parameter $\beta$ (a)PST case (b) PHF case, on temperature profiles at Mn=1, $\mathrm{B}^{*}=-0.01, \mathrm{~A}^{*}=-0.01 \mathrm{e}=1$, and $\operatorname{Pr}=1$, and $\gamma=\mathbf{1}$

Figure2 demonstrates the effect of the slip parameter $\gamma$ on the axial velocity $f^{\prime}(\eta)$. It is readily seen the $\gamma$ has strong effect on the flow field. In fact, the amount of slip $1-\mathrm{f}^{\prime}(0)$ increases monotonically with $\gamma$ from the no-slip situation for $\gamma=0$ and towards full slip as $\quad \gamma \rightarrow \infty$. The latter limiting case implies that the frictional resistance between the cooling liquid and the stretching sheet is eliminated and the stretching of the sheet does no longer impose any motion of the cooling liquid. The effect of the magnetic parameter $M_{n}$ on the axial velocity $f^{\prime}(\eta)$ is shown in figure 3. From this figure, it is evident that axial velocity decreases with increasing values of the magnetic parameter $M_{n}$. That is, the Lorentz force offers resistance to the fluid flow. Figure 4(a),(b) project the effects of the magnetic parameter $M_{n}$ on the temperature field in the PST and PHF cases. These plots highlight the fact that increasing value of $M_{n}$ result in increasing the thermal boundary-layer thickness. The effect of the Prandtl number Pr on heat transfer can be analyzed from figure 5(a),and(b) in the PST and PHF cases. In this graph we have seen that an increase in Prandtl number results in a decrease of the temperature distribution in both cases under consideration. The effect of the slip temperature $\gamma$ on temperature field is depicted in figures 6(a), and (b) in the cases of PST and PHF. From these plots it is observed that increasing values of the slip parameter $\gamma$ results in thickening of the boundary layer. The effect of the variable thermal conductivity parameter $\varepsilon$ on the temperature field is shown in fiure. 7(a) and (b) is the cases of PST and PHF. Increasing values of the variable thermal conductivity parameter $\varepsilon$ results in thickening of the thermal boundary layer. Figures 8(a),and 8(b) depict the effects of the space-dependent heat source/sink parameter A*. It is observed that the energy is generated in the thermal boundary layer, causing the temperature profiles to increase with the increase with the increasing values of $A^{*}>0$, whereas in the case of $A^{*}<0$ the energy is being observed in the boundary layer as a result the temperature falls considerably with decreasing values of $A^{*}$. The effect of the temperature-dependent heat source/sink parameter $\mathrm{B}^{*}$ on temperature field is shown in figures 9(a), and 9(b). The influence of the Casson parameter on the velocity and temperature (PHF, PST cases) profiles is shown in figures 10 and figure 11 (a), and 11(b) respectively. The magnitude of the velocity is greater in the case of Carson fluid when compared with a viscous fluid. Hence, in general, with an increase in $\beta$, the velocity of the fluid decreases for a stretching sheet. Figure 12(a),(b) in the cases of PST and PHF, respectively shown the effect of Casson parameter on the temperature profile. The temperature profile increase with $\beta$. 
Table I. Skin-friction coefficient for various values of $\gamma$ when $M_{n}=0$ and $\beta=\infty$

\begin{tabular}{|c|c|c|c|}
\hline$\gamma$ & Sahoo [43] & Mahantesh [47] & Present result \\
\hline 0.0 & -1.0000 & -1.0000 & -1.0000 \\
\hline 0.1 & -0.8721 & -0.87208 & -0.87200 \\
\hline 0.2 & -0.7764 & -0.77638 & -0.77635 \\
\hline 0.5 & -0.5912 & -0.59120 & -0.59120 \\
\hline 1 & -0.4302 & -0.43016 & -0.43014 \\
\hline 2 & -0.2840 & -0.28398 & -0.28395 \\
\hline 5 & -0.1448 & -0.14484 & -0.14480 \\
\hline 10 & -0.0812 & -0.08124 & -0.08120 \\
\hline 20 & -0.0438 & -0.4379 & -0.4375 \\
\hline 50 & -0.0186 & -0.01860 & -0.01858 \\
\hline
\end{tabular}

Table 2.Nusselt number for PST and PHF cases for different values ofgoverning parameters when $\beta=\infty$

\begin{tabular}{|l|l|l|l|l|l|l|l|l|l|}
\hline$\gamma$ & $\mathbf{M}_{\mathbf{n}}$ & $\mathbf{P r}$ & $\mathbf{A}^{*}$ & \multicolumn{1}{|c|}{$\mathbf{B}^{*}$} & $\boldsymbol{\varepsilon}$ & $\begin{array}{c}\text { Values of } \\
\text { PST }\end{array}$ & $\begin{array}{c}\theta^{\prime}(\mathbf{0}) \\
\text { PHF }\end{array}$ & \multicolumn{2}{|c|}{ Mahantesh [47] } \\
\hline & & & & & & & & $\mathbf{P S T}$ & PHF \\
\hline 0.0 & 1.0 & 1.0 & -0.01 & -0.01 & 0.1 & 0.836978 & 0.866430 & 0.836973 & 0.866428 \\
1.0 & & & & & & 0.518720 & 0.755690 & 0.518719 & 0.755687 \\
5.0 & & & & & & 0.271048 & 0.497640 & 0.271043 & 0.497638 \\
\hline 1.0 & 1.0 & 1.0 & -0.01 & -0.01 & 0.1 & 0.518720 & 0.755690 & 0.518717 & 0.755687 \\
& 2.0 & & & & & 0.410480 & 0.656625 & 0.410482 & 0.636622 \\
& 3.0 & & & & & 0.344735 & 0.577456 & 0.344737 & 0.377458 \\
\hline 1.0 & 1.0 & 1.0 & -0.01 & -0.01 & 0.1 & 0.518720 & 0.755686 & 0.518719 & 0.755687 \\
& & 2.0 & & & & 0.832307 & 0.877665 & 0.832306 & 0.877669 \\
& & 5.0 & & & & 1.478850 & 0.963913 & 1.478850 & 0.163913 \\
\hline 1.0 & 1.0 & 1.0 & -0.5 & -0.01 & 0.1 & 0.912442 & 0.896505 & 0.912443 & 0.896506 \\
& & & 0.0 & & & 0.502510 & 0.739318 & 0.502414 & 0.739313 \\
& & & 0.3 & & & 0.385214 & 0.485420 & 0.385212 & 0.501422 \\
\hline 1.0 & 1.0 & 1.0 & -0.01 & -0.5 & 0.1 & 0.912448 & 0.896503 & 0.912443 & 0.896506 \\
& & & & 0.0 & & 0.502515 & 0.739315 & 0.302314 & 9.739913 \\
& & & & 0.3 & & 0.384525 & 0.485420 & 0.384521 & 0.485424 \\
\hline 1.0 & 1.0 & 1.0 & -0.01 & -0.01 & -0.5 & 0.995745 & 2.221570 & 0.995746 & 2.221570 \\
& & & & & 0.1 & 0.345258 & 0.755187 & 0.345255 & 0.755687 \\
& & & & & 0.5 & 0.128426 & 0.221566 & 0.128425 & 0.221566 \\
\hline
\end{tabular}

\section{References}

[1] Sakidas B C (1961) Boundary-layer behaviour as continuous solid surface:I. Boundary-layer equation for two dimensional and axisymmetric flow. AIChEJ7: 26-28

[2] Sakiadis B C (1961) Boundary layer behaviour on continuous solid surface : II. Boundary layer on a continuous flat surface.AIChE 7(2): 221-225

[3] BlasiusH (1098) Gronzschichten in FlussigkeitenmitkleinerReibung. Z Math Phys 56 : 1-37

[4] Crane L J (1970) Flow past a stretching sheet. Z Angew Math Phys 21 : 645-647

[5] CarragherP,Crane L J (1982) Heat transfer on a continuous stretching sheet. Z Angew Math Mech 62: $564-565$

[6] Gupta P S, Gupta A S (1977) Heat and mass transfer on a stretching sheet with suction or blowing. Can J ChemEng 55 : $744-746$

[7] Grabka L J, Babba K M (1989) Heat transfer characteristics of a continuous stretching surface with variable temperature. ASME J Heat transfer $107: 248-250$

[8] Chen C K, CharM I (1988) Heat transfer of a continuous stretching surface with suction or blowing. J. Math Anal Appl 133 : $568-580$

[9] Chiam T C (1997) Magnetohydrodynamic heat transfer over a non-isothermal stretching sheet. ActaMech 122: 169-179

[10] Cortell R (2005) Flow and heat and mass transfer of a fluid through a porous medium over a stretching surface with internal heat generation/ absorption and suction/blowing. Fluid Dyn Res 37 : 231-245

[11] Cortell R (2007) Viscous flow and heat transfer over a non-linearly stretching sheet. Appl Math Comput 184: 864-873

[12] Cortell R (2011) Heat and fluid flow due to non-linearly stretching surfaces. ApplMathsComput 217- 7564-7572

[13] Sarpakaya T (1961) Flow of Non-Newtonian fluids in magnetic field AIChEJ 7 : 324-328

[14] Pal D.Mondal H (2010) Effect of variable viscosity on MHD non-Darcy mixed convective heat transfer over a stretching sheet embedded in a parameter medium with non-uniform heat source/sink. Commun Nonlinear Sci Number Simul 15: 1553-1564

[15] Pol D. Mondal H (2010) Hydromagnetic non-Darcy flow and heat transfer overa stretching sheet in the presence of thermal radiation and Ohmic dissipation. Commun Nonlinear SciNumerSimul 15: 1197-1209 
[16] HyatT,Abbas Z, Pop I, Asyhur (2010) Effects of radiation and magnetic field on the mixed convection stagnation-point flow over a vertical stretching sheet in a porous medium. Int J Heat Mass Transfer53: 466-479

[17] Nondeppanavar M M, Vajravelu K. Abel M S (2011) Heat transfer in MHD viscoelastic boundary layer flow over a stretching sheet with thermal radiation and Non uniform heat source/sink. Commu Non-linear sciNumersimul 16: 3578-3590

[18] Chakraborti A, Gupta A S (1979) Hydromagnetic flow and heat transfer over a stretching sheet. Q Appl Math 37 : $73-78$

[19] Andersson H I, Bech K H, Dandapat B S(1992) Magnetohydrodynamic flow of a power-law fluid over a stretching sheet. Int J Nonlinear Mech 27: 929-936

[20] Datti P S, Prasad K V, Abel M S, Joshi A (2004) MHD visco-elastic fluid flow over a non-isothermal stretching sheet. Int J EngSci 42: 935-946

[21] Abel M S, Khan S K, Prasad K V (2002) Study of viscoelastic fluid and heat transfer over a stretching sheet with variable viscosity. Int J Non-Linear Mech 37(1): 81

[22] Abel S, Joshi A, Sonth R M (2001) Heat transfer in a MHD visco-elastic fluid over a stretching surface. Z Angew Math Mech 81: 691698

[23] Vajravelu K, Rollins D (1972) Heat transfer in electrically conducting fluid over a stretching surface. Int J Non-Linear Mech 27(2): 265-277

[24] Vajravelu K, Nayfeh J (1993) Convective heat transfer at a stretching sheet. ActaMech 116: 47-54

[25] Vajravelu K, Cannon J R (2006) Fluid flow over a non-linear stretching sheet. Appl Math Comput 81: 609-618

[26] Bhattacharya K. Vajravelu K (2012) Stagnation-point flow and heat transfer over an exponentially shrinking sheet. Commun Nonlinear SciNumerSimul 17: 2728-2734

[27] Abo-Eldahab E M, E I Aziz M A (2004) Blowing/suction effect on hydromagnetic heat transfer by mixed convection from an inclined continuously stretching surface with internal heat generation/absorption. Int J ThermSci 43: 709-719

[28] Nundeppanavar M M, Vajravelu K, Abel M S, Ng C O (2011). Heat transfer over a non-linear stretching sheet with non-uniform heat source and variable wall temperature. Int J Heat Mass transf. 54: 4960-4965.

[29] Abel M S, Siddheshwar P H, Nandeppanavar M M (2007) Heat transfer in a viscoelastic fluid past a stretching sheet with non-uniform heat source. 50: 960-966.

[30] CortellBataller R (2007) Viscoelastic fluid flow and heat transfer over a stretching sheetunder the effects of non-uniform heat source, viscous dissipation and thermal radiation. Int J Heat Mass Transf 50: 3152-3162

[31] Navier CLMH (1827) Sur les. lois du movement des fluids Men Acad R SciIntFrance 6: 389-440

[32] Beavers G S, Joseph D D (1967) Boundary condition at a naturally permeable wall. J Fluid Mech 30: 197-207

[33] Laplace P, Arquis E (1998) Boundary layer over a slotted plate.Eur J Mech B, Fluids 17 : 331-335.

[34] Miksis M J, Davis S H (1994) Slip over rough and coated surface. J Fluid Mech 273: 125-139

[35] God-el-Hak M (1999) The fluid mechanics of microdevices - the freeman scholar lecture. ASME J Fluids Eng 121: 5-33.

[36] Andersson H I (2002) Slip flow past a stretching surface. ActaMech 158: 121-125

[37] Malipatil S B (2011) Mathematical Analysis on some aspects of momentum, heat and mass transfer in viscous and viscoelastic boundary layer flow over stretching source embedded in porous medium. Ph.D. thesis, Gulbarga University, Gulbarga - 585106, Karnataka

[38] Ariel P D, Hayat T, Asghar S (2006) The flow of an elastico-viscous fluid past a stretching sheet with partial slip. ActaMech 187: 2935

[39] Hayat T, Javed T, Abbas T (2008) Slip flow and heat transfer of a second grad fluid past a stretching sheet through a porous space. Int J Heat Mass Transf 51: 4528-4534

[40] Andersson H I (1995) A exact solution of the Navier-Stokes equations for magnetohydrodynamics flow. ActaMech 113: 241-245

[41] Pavlov K B (1979) Magnetohydrodynamic flow of an incompressible viscous fluid caused by deformation of a plane surface. MynGidrodin 4: 146-147

[42] Abramavitz M, Stegum L A (1972) Handbook of Mathematical functions. National Bureau of standards/Ann Math Soc Providence, p.55

[43] Wang C Y (2002) Flow due to a stretching boundary with partial slip - an exact solution of the Navier-Stokes equation. ChemEngSci 57: $3741-3747$

[44] Sahoo B (2011) Flow and heat transfer of a non-Newtonian fluid past a stretching sheet with partial slop. Commun Non-linear SciNumerSimul 15: 602-615

[45] Mustafa A, Mahamad A, Meyahed A M (2012). Non-uniform heat generation effect on heat transfer of a non-Newtonian power law fluid over a non-linear stretching sheet. Meecanica dui 10.1007/51102-011-9499-9

[46] Markin J K, Kumarin V (2012) The unsteady heat transfer due to a heat source in an MHD stretching sheet flow. Meccanica. doi: 10.1007/S11012-012-95562

[47] Mahantosh M, Nandepanavar K. Vajravelu M, Subhas Abel, M N Siddalingappa (2013). MHD flow and heat transfer over a stretching surface with variable thermal conductivity and partial slip.Meccanicdoi: 10.1007/S11012-012-9677-4 